but Gould and $\mathrm{Gragg}^{9}$ have recently suggested some guidelines. The diagnosis must be correct, so that the first essential has to be a physical examination to exclude organic disease and the possibility of true infestation. Wilson ${ }^{3}$ described one woman with a bizarre story compatible with a delusional state who was found to have pediculosis capitis. Gould and Gragg ${ }^{9}$ advise listening to the patient without argument, attempting to build up trust and a positive bond. They advocate trying to reduce the patient's sense of isolation and the use of psychoactive drugs to lessen the patient's anxiety.

Whether treated by dermatologist or psychiatrist the prognosis for recovery is poor, and some patients have remained deluded for 20 years. $^{1}$

\footnotetext{
${ }^{1}$ Wilson, J W, and Miller, H E, Archives of Dermatology and Syphilology, $1946,54,39$.

2 Schrut, A H, and Waldron, W G, fournal of the American Medical Association, 1963, 186, 429.

3 Wilson, J W, Archives of Dermatology and Syphilology, 1952, 66, 577.

4 Tullett, G L, British fournal of Dermatology, 1965, 77, 448.

5 Evans, P, and Merskey, H, British fournal of Medical Psychology, 1972, 45,

6 Hopkinson, G, Psychiatria Clinica, 1973, 6, 330.

7 Aleshire, I, fournal of the American Medical Association, 1954, 155, 15.

* Pope, F M, Practitioner, 1970, 204, 421.

${ }^{9}$ Gould, W M, and Gragg, T M, Archives of Dermatology, 1976, 112, 1745.
}

\section{Difficulties in leukaemia diagnosis}

Early attempts at chemotherapy in leukaemia suggested that the response of acute lymphoblastic leukaemia (ALL) was better than that of acute nonlymphoid leukaemia. Since then, diagnosis has been shown to be vital in determining both prognosis and treatment. While many acute leukaemias can be typed, ${ }^{12}$ there remains a group sometimes described (inappropriately) as acute undifferentiated leukaemia where exact diagnosis is difficult. In these cases the blast cells may be primitive precursors of myeloblasts or lymphoblasts, and they are difficult to recognise, though cytochemical procedures ${ }^{3}$ may help to separate them. Bennett $e t a^{1}$ found that some of the blast cells in all patients with myeloid leukaemia stained positively with myeloperoxidase or Sudan Black B, the positive reaction being particularly helpful in distinguishing this group from patients with lymphoblastic leukaemia. They also found help from fluoride-sensitive non-specific esterase stains, which give a positive reaction in most monocytic, granulocytic, and myelomonocytic leukaemias. Monocytoid cells usually show a very strong reaction which is inhibited by sodium fluoride. In cells of myeloid origin, on the other hand, the reaction is unaffected by fluoride and in lymphoblasts the reaction is negative.

Simple cytochemistry may, then, help in the diagnosis of all acute nonlymphoid leukaemias but may give only negative help in ALL, in which identifying antigens on the cell surface of lymphoblasts ${ }^{4-6}$ is the key. While some $70 \%$ of the blast cells do not carry B- or T-cell markers, they do react with antisera specific for ALL; and, of the rest, $28 \%$ carry Tlymphocyte markers and rare patients (about 3\%) have blasts of B-cell origin.

Recently a small but important group of patients has been identified who present with the clinical and morphological features of ALL, but in whom further evidence suggests that the correct diagnosis is chronic myeloid leukaemia (CML) presenting in blast cell crisis without any previous detectable chronic phase. ${ }^{7}$ These patients have minor changes in the peripheral blood not seen in ALL, including the Pelger-Huët anomaly in the shape of nuclei, hypogranular neutrophils, and primitive monocytoid cells. In fact, the true diagnosis has been established only when the Philadelphia chromosome has been identified.

The Philadelphia chromosome is present in about $90 \%$ of patients with CML and may be regarded as a specific marker for the disease, as it occurs only rarely in other conditions. Janossy et $a l^{8}$ examined cell surface markers in 14 patients with the blast cell transformation of CML and showed that some blast cells reacted with a specific ALL antiserum of non-T and non-B cell type and that all showed diminished expression of cholera toxin receptors when compared with granulocytes from the chronic phase of CML. They suggested that the blast cell crisis of CML is morphologically heterogeneous and that in some patients cells develop which are morphologically identical to those seen in most patients with ALL and which cannot be differentiated from ALL blasts by the use of membrane markers.

These findings have practical importance, since the blast cell crisis of CML is often resistant to treatment whereas adult ALL has a better prognosis. Similar clinical and morphological observations have recently been reported in children. ${ }^{9} \mathrm{CML}$ in children is rare, but clearly there have been examples of children presenting in the blast cell phase of CML who were misdiagnosed as having ALL. The much improved prognosis for children with ALL makes accurate diagnosis necessary, and since there is evidence of other karyotypic abnormalities in AML which may be related to poor prognosis ${ }^{\mathbf{1 0}}$ there is a strong case for examining chromosomes in all patients with acute leukaemia. Certainly chromosome analysis should be undertaken in any patient whose blast cells cannot be classified precisely or in any child with ALL who does not enter an early remission.

Leukaemic states described variously as "smouldering leukaemia," "preleukaemia," or "subacute leukaemia" are probably better defined" as "refractory anaemia with excess of blasts" but are difficult to diagnose. Nevertheless, they are usually associated with dyserythropoietic changes in the bone marrow and sometimes with the presence of ring sideroblasts. The peripheral blood may show neutropenia, hypogranular polymorphs, the Pelger-Huët anomaly, and low monocyte counts in association with a hypercellular bone marrow with a varying degree of abnormal granulocyte maturation. The blast cell count is usually $10-20 \%$, and leukaemia can usually be diagnosed with confidence only by showing a progressive increase in blast cells in the blood and bone marrow at repeated examinations.

Recent studies in patients with lymphoid neoplasms have also helped the classification of leukaemia including the separation of several disease states previously collected under the label of chronic lymphatic leukaemia (CLL). Leukaemic reticuloendotheliosis, ${ }^{11}$ sometimes called hairy cell leukaemia, presents at a younger age than CLL with progressive tiredness, moderate to massive splenomegaly, and pancytopenia with variable numbers of abnormal mononuclear cells in the blood. The lymph nodes are usually enlarged, but are seldom prominent. The abnormal cells seen in variable numbers in the peripheral blood and bone marrow may be mistaken for the lymphocytes of CLL. They are distinguished by their abundant cytoplasm; stippled chromatin, in which nucleoli can often be discerned; and particularly by their irregular "hairy" 
cytoplasmic edges. Cytochemically they stain with acid phosphatase, and this reaction is tartrate-resistant. Electron microscopy gives absolute confirmation of the diagnosis. Leukaemic reticuloendotheliosis should be distinguished from other lymphoproliferative disorders since splenectomy improves the pancytopenia and prolongs survival.

Prolymphocytic leukaemia ${ }^{12}$ is a rare variant of CLL occurring predominantly in elderly men. It is characterised by tiredness, weight loss, sweats, and fevers. There is massive splenic enlargement, moderate hepatic enlargement, but little or no lymphadenopathy. The blood usually contains very many (occasionally over 1 million/ $\mu \mathrm{l}$ ) large lymphocytes with a moderate amount of cytoplasm and well-condensed nuclear chromatin with a single prominent nucleolus. The prognosis is poor, and treatment which is usually effective in CLL is unhelpful. The place of splenectomy remains uncertain. Both leukaemic reticuloendotheliosis and prolymphocytic leukaemia are not difficult to separate from lymphosarcoma cell leukaemia, which occurs terminally in $5-10 \%$ of patients with lymphosarcoma. It is the rare patient with lymphosarcoma who presents with leukaemia who causes difficulty. In such cases, however, the lymphocyte count is usually lower than in CLL or prolymphocytic leukaemia and characteristically the cells have a primitive nuclear chromatin pattern with nuclear clefts and indentations.

Perhaps the clearest indication for attempting to define different types of leukaemia more exactly is the clinical importance of accurate diagnosis. The different courses and responses to treatment of ALL, acute non-lymphoid leukaemia, and the chronic leukaemias are now clear. But it remains to be seen whether the subdivision of acute nonlymphoid leukaemia will result in more rational treatment.

1 Bennett, J M, et al, British fournal of Haematology, 1976, 33, 451.

2 Mathé, G, and Rappaport, H, Histological and Cytological Typing of Neoplastic Diseases of Haematopoietic and Lymphoid Tissues. Geneva, World Health Organisation, 1975.

${ }^{3}$ Hayhoe, F G J, and Flemans, R J, An Atlas of Haematological Cytology. London, Wolfe Medical Books, 1969.

4 Brouet, J C, et al, British fournal of Haematology, 1976, 33, 319.

5 Seligmann, M, British fournal of Haematology, 1975, 31, (suppl), 1.

6 Greaves, M F, et al, Clinical Immunology and Immunopathology, 1975, 4, 67.

${ }^{7}$ Beard, M E J, et al, British fournal of Haematology, 1976, 34, 167.

8 Janossy, G, et al, British fournal of Haematology, 1976, 34, 179.

- Secker-Walker, L M, et al, Lancet, 1976, 2, 1405.

10 Golomb, H M, Vardiman, J, and Rowley, J D, Blood, 1976, 48, 9.

11 Catovsky, D, et al, British fournal of Haematology, 1974, 26, 9.

12 Galton, D A G, et al, British fournal of Haematology, 1974, 27, 7.

\section{Destructive fibrosis}

The formation of fibrous tissue is the end result of the process of repair by which wounds are healed. As such, it is of enormous survival value to the individual; yet it may also lead to serious complications. The contracture of a scar on the skin may have cosmetic and functional effects. Even more disfiguring is the common keloid, in which there is an overgrowth of fibrous tissue severe enough at times to simulate a benign tumour. The fibrosis attending a tendon injury may lead to adhesions to the surrounding sheath with impairment of movement. A common fibrotic process of the palmar fascia is Dupuytren's contracture, which starts as a thickening of the fascia and proceeds to a deforming indrawing of the fingers (usually the ring and little fingers) towards the palm. Sometimes the plantar fascia is also affected, and rarely fibrous plaques may develop in the corpora cavernosa of the penisPeyronie's disease. An extremely disabling type of destructive fibrosis affects the joints in chronic arthritis, especially in rheumatoid disease, in which ankylosis leads to severe deformity and crippling.

When hollow muscular viscera such as the oesophagus, bowel, or urethra undergo fibrous repair after trauma or inflammatory destruction by ulceration the end result may be progressive stenosis with proximal obstruction. In a similar way the heart valves may undergo contracture with stenosis after the inflammatory destruction of acute rheumatic fever. Syphilitic disease of the aortic valves leads to severe incompetence, and the destruction of the aorta may proceed to formation of an aneurysm. Another serious fibrotic heart lesion is constrictive pericarditis, which interferes with the proper filling of the heart. The usual causes are pyogenic or tuberculous pericarditis or the pericarditis that may occur in rheumatoid disease. When there is massive or progressive destruction of the liver parenchyma, the proliferation of fibrous tissue leads to cirrhosis, which may then strangle the venous circulation and lead to portal hypertension.

In progressive systemic sclerosis (scleroderma) there is a more generalised fibrosis, which may affect the oesophagus, bowel, lungs, and heart, as well as the skin. The obscure retroperitoneal fibrosis, which sometimes entraps the ureters and leads to renal failure, is sometimes associated with treatment with methysergide given for the prophylaxis of migraine. Two other chemical causes of widespread fibrosis are practolol (which leads to a severe peritoneal fibrosis that may cause intestinal obstruction) and vinyl chloride monomer (which may produce an industrial form of progressive systemic sclerosis together with osteolysis and occasional hepatic angiosarcoma). Two inhaled substances lead to pulmonary fibrosis: silica produces circumscribed nodules, especially in the upper parts of the lungs, whereas asbestos induces a diffuse fibrosis affecting chiefly the bases.

The Arthritis and Rheumatism Council recently organised a symposium on the fibrotic process, in which many aspects of normal and destructive fibrosis were discussed. ${ }^{1}$ While little is known about the factors leading to such common lesions as the keloid and Dupuytren's contracture, the role of the macrophage, especially when it is damaged by silica or asbestos, in elaborating a fibrogenic factor is becoming clearer. In progressive systemic sclerosis, vinyl-chloride disease, and silicosis there are immunological factors, at present ill-defined, which appear to augment the fibrotic process.

Several agents have been used for treating destructive fibrosis but on the whole their effects have not been impressive. Corticosteroids inhibit fibroblasts in vitro and osteoblasts in vivo, but they do not appear to inhibit inflammatory fibrosis in the joints or the heart. Another group of drugs, called lathyritic agents, interfere with the cross-linkage and stabilisation of newly formed collagen; and, of these, penicillamine is the most useful and least toxic, but it is effective in the treatment of progressive systemic sclerosis only in the active phase of the disease, when the biosynthesis of collagen is taking place. Two other possible therapeutic approaches are the use of proline analogues that interfere with the formation of hydroxyproline, an essential amino-acid of collagen, and agents that interact with the microtubules of the fibroblast, which play a vital part in collagen secretion. The results of experimental work on proline analogues are interesting, but the probable toxicity of the substances used makes them unlikely 